\title{
Effects of curcumin on cancer cell mitochondrial function and potential monitoring with ${ }^{18} \mathrm{~F}$-FDG uptake
}

\author{
KYUNG-HO JUNG $^{1,2^{*}}$, JIN HEE LEE $^{1,2^{*}}$, JIN WON PARK $^{1,2}$, SEUNG-HWAN MOON $^{1}$, \\ YOUNG SEOK CHO ${ }^{1}$, YEARN SEONG CHOE ${ }^{1,2}$ and KYUNG-HAN LEE ${ }^{1,2}$ \\ ${ }^{1}$ Department of Nuclear Medicine, Samsung Medical Center, and ${ }^{2}$ Samsung Advanced Institute for Health \\ Sciences and Technology, Sungkyunkwan University School of Medicine, Seoul, Republic of Korea
}

Received September 23, 2015; Accepted November 10, 2015

DOI: $10.3892 /$ or. 2015.4460

\begin{abstract}
A better understanding of how curcumin influences cancer cell biology could help devise new strategies to enhance its antitumor effect. Many curcumin actions are proposed to occur by targeting mitochondrial function, among which glucose metabolism and reactive oxygen species (ROS) production are pivotal. However, little is known of how curcumin influences cancer cell glucose metabolism. We thus evaluated the effect of curcumin on cancer cell glucose metabolism and mitochondrial function, and further investigated whether these responses could be modified to enhance the anticancer potency of the compound. MCF-7 breast cancer cells treated with curcumin were measured for ${ }^{18} \mathrm{~F}$-fluorodeoxyglucose $\left({ }^{18} \mathrm{~F}-\mathrm{FDG}\right)$ uptake, lactate production, hexokinase activity, oxygen consumption rate (OCR), ROS production and mitochondrial membrane potential (MMP). Activation of signaling pathways was evaluated by western blots, and cell survival was assessed with sulforhodamine B assays. Curcumin stimulated a 3.6-fold increase of ${ }^{18} \mathrm{~F}$-FDG uptake in MCF-7 cells, along with augmented hexokinase activity and lactate efflux. This was accompanied by significantly suppressed cellular OCR, consistent with a metabolic shift to glycolytic flux. Inhibiting this metabolic response with 2-deoxyglucose (2-DG) blocked curcumin-induced mTOR activation and resulted in a greater anti-proliferative effect. Curcumin-induced MMP depolarization led to reduced ROS production, which may hinder the anticancer effect of the compound. Intracellular ROS was completely restored by adding $\mathrm{Cu}^{2+}$, which can bind and modify the curcumin's physico-chemical property, and this resulted in a marked potentiation of its anti-proliferative effect. Thus, curcumin suppresses cancer cell MMP and ROS genera-
\end{abstract}

Correspondence to: Professor Kyung-Han Lee, Department of Nuclear Medicine, Samsung Medical Center, 50 Ilwondong, Gangnamgu, Seoul, Republic of Korea

E-mail:khnm.lee@samsung.com

*Contributed equally

Key words: curcumin, cancer, glycolysis, mitochondria, reactive oxygen species, 2-DG, copper tion, and this response is accompanied by stimulated ${ }^{18} \mathrm{~F}-\mathrm{FDG}$ uptake via shifting of metabolism from mitochondrial respiration to glycolytic flux. These mitochondrial and metabolic responses may provide potential targets that can help enhance the anticancer action of curcumin.

\section{Introduction}

Curcumin, a biocompatible polyphenol of the dietary spice turmeric, has been shown to exhibit anticancer effects in multiple cancers including those of the breast, ovary, colon and brain (1). However, the clinical application of curcumin has been hindered to date, not only due to its low solubility and bioavailability, but also by limitations in its anticancer potency. It is, therefore, important to better understand how curcumin influences the biology of cancer cells so that new strategies can be devised to enhance its antitumor effect.

Recent evidence points to mitochondria as the target for many of the beneficial effects of curcumin (2). A major function of mitochondria is to generate energy by utilizing substrates. Although malignant cells undergo reprogramming of energy metabolism toward aerobic glycolysis (3), they also depend on mitochondrial oxidative metabolism for proliferation and survival. Thus, cancer cell glucose metabolism is gaining interest as a promising target for cancer treatment (4), and is exploited by positron emission tomography (PET) with ${ }^{18} \mathrm{~F}$-fluorodeoxyglucose $\left({ }^{18} \mathrm{~F}\right.$-FDG) for monitoring treatment efficacy (5). Another important mitochondrial function is the production of reactive oxygen species (ROS), which mediates crucial cellular tasks including signal transduction and induction of apoptosis (6). Hence, cancer-specific modifications to ROS and anti-oxidants are also considered a potential target for therapy (6).

A link between curcumin action and glucose metabolism is suggested by observations in skeletal muscle (7) and myotubes (8) that curcumin can stimulate glucose uptake. However, how cancer cell glucose metabolism is influenced by curcumin, and whether suppressing this response affects its anticancer action has not been investigated. Furthermore, although curcumin is through to induce apoptosis of cancer cells through oxidative stress $(9,10)$, it is not known whether suppressing the ROS scavenging activity of curcumin (11) can enhance its antitumor effect. This could be relevant for 
the development of newer curcumin analogs with strengthened pro-oxidant properties for improved therapeutic response $(12,13)$. In addition, since cancer cells heavily rely on glucose utilization, understanding how metabolism-targeting drugs modulate tumor ${ }^{18} \mathrm{~F}$-FDG uptake may not only help uncover new strategies for cancer treatment but could also expand the role of PET imaging for monitoring treatment response.

In the present study, we examined how curcumin influences cancer cell glucose metabolism and mitochondrial function, including ${ }^{18} \mathrm{~F}$-FDG uptake, lactate production, hexokinase activity, oxygen consumption, mitochondrial membrane potential (MMP) and ROS production. We further investigated whether restricting glycolysis with 2-deoxyglucose (2-DG) and addition of $\mathrm{Cu}^{2+}$, known to reduce curcumin's anti-oxidant property (14-16), can enhance the anticancer action of the compound.

\section{Materials and methods}

Cancer cell lines and culture. MCF-7 human breast cancer and CT26 colon cancer cell lines were from the American Type Culture Collection (ATCC; Rockville, MD, USA), and maintained in MEM (MCF-7 cells) or RPMI-1640 media (CT26 cells) supplemented with $10 \%$ fetal bovine serum and $1 \%$ penicillin/streptomycin in a humidified incubator at $37^{\circ} \mathrm{C}, 5 \%$ $\mathrm{CO}_{2}$. Cells grown in a monolayer were routinely split 2 times a week, and experiments were performed at $48 \mathrm{~h}$ after seeding. For cell treatment, stock solutions of curcumin dissolved in DMSO, 2-DG dissolved in phosphate-buffered solution (PBS), and $\mathrm{CuSO}_{4}$ dissolved in $0.05 \mathrm{M}$ Tris- $\mathrm{HCl}(\mathrm{pH}$ 7.4) were used.

Reagents and antibodies. Curcumin, 2-DG, copper sulfate (II) $\left(\mathrm{CuSO}_{4}\right)$, oligomycin, carbonyl cyanide-4-(trifluoromethoxy) phenylhydrazone (FCCP) and antimycin-A were from SigmaAldrich Chemical Co. (St. Louis, MO, USA). CM-H ${ }_{2}$ DCFDA (5-(and-6)-chloromethyl-29,79-dichlorodihydrofluorescein diacetate, acetyl ester) and MitoTracker Red FM was from Invitrogen Life Technologies (Grand Island, NY, USA). Antibodies against phosphorylated mammalian target of rapamycin (p-mTOR) and phosphorylated extracellular-signalregulated kinase (p-ERK), and anti-rabbit and anti-mouse secondary antibodies were from Cell Signaling Technology (Danvers, MA, USA). Polyclonal antibody against $\beta$-actin was from Santa Cruz Biotechnology (Dallas, TX, USA). Rabbit polyclonal antibody against glucose transporter 1 (GLUT1) was from Abcam (Cambridge, MA, USA).

Sulforhodamine $B$ (SRB) assay. Surviving cell content following treatment was evaluated by SRB assays. Briefly, cells seeded overnight on a 96-well plate were treated for indicated durations of time, and cell monolayers were fixed with $10 \%$ (wt/vol) trichloroacetic acid at $4^{\circ} \mathrm{C}$. After cells were stained with SRB dye for $30 \mathrm{~min}$, excess dye was removed by washing repeatedly with $1 \%$ (v/v) acetic acid. Protein-bound dye was finally dissolved in $10 \mathrm{mM}$ Tris base solution and subject to spectrophotometric measurement of absorbance at $510 \mathrm{~nm}$ using a microplate reader.

FDG uptake measurement. Cells in 12-well plates were incubated with $370 \mathrm{kBq}(10 \mu \mathrm{Ci})$ of the radiolabeled glucose analogue ${ }^{18} \mathrm{~F}-\mathrm{FDG}$ for $40 \mathrm{~min}$ at $5 \% \mathrm{CO}_{2}, 37^{\circ} \mathrm{C}$. After rapid washing twice with cold PBS, cells were lysed with $500 \mu \mathrm{l}$ of $0.01 \mathrm{~N} \mathrm{NaOH}$ and cell-associated radioactivity was measured on a high-energy $\gamma$-counter (Perkin-Elmer, Waltham, MA, USA). Radio-uptake levels were corrected for cell content as assessed by Bradford protein assays.

Lactate production. L-lactate production was measured from $100 \mu \mathrm{l}$ of culture medium using a Cobas assay kit (Roche/ Hitachi) following the manufacturer's instructions. In the assay, lactate is enzymatically converted to pyruvate and hydrogen peroxide. Hydrogen peroxide then undergoes an enzymatic reaction to generate a colored dye that is measured by absorbance on a microplate spectrophotometer. Lactate concentration was calculated from a standard curve of serially diluted standards and expressed in $\mathrm{mU} / \mathrm{mg}$.

Hexokinase activity. Cells homogenized in homogenizing buffer $\left(50 \mathrm{mM}\right.$ triethanolamine and $\left.5 \mathrm{mM} \mathrm{MgCl}_{2} ; \mathrm{pH} 7.6\right)$ were centrifuged at $1,000 \mathrm{x} \mathrm{g}$ for $5 \mathrm{~min}$ at $4^{\circ} \mathrm{C}$. The pelleted mitochondrial fraction was isolated and $50 \mu \mathrm{l}$ was mixed with $2.52 \mathrm{ml}$ of reaction buffer containing $39 \mathrm{mM}$ triethanolamine, $216 \mathrm{mM}$ D-glucose, $0.74 \mathrm{mM}$ adenosine 5'-triphosphate, $7.8 \mathrm{mM}$ magnesium chloride, $1.1 \mathrm{mM} \beta$-nicotinamide adenine dinucleotide phosphate, and 2.5 units of glucose 6-phosphate dehydrogenase. The reaction mixture was repeatedly measured at $25^{\circ} \mathrm{C}$ for spectrophotometric absorbance at $340 \mathrm{~nm}$. Hexokinase activity was expressed as $\mathrm{mU} / \mathrm{mg}$ of protein, and one unit of activity was defined as the amount of hexokinase activity that phosphorylates $1 \mu \mathrm{mol}$ of glucose per min at $25^{\circ} \mathrm{C}$.

Oxygen consumption rate (OCR). Cells were seeded in $100 \mu \mathrm{l}$ of MEM media at a density of $5 \times 10^{4}$ cells/well on analyzer plates. A Seahorse XF24 Extracellular Flux analyzer (Seahorse Bioscience Inc., North Billerica, MA, USA) with a solid state sensor probe analyzed the concentration of oxygen dissolved in the media at 2-sec intervals. Following measurement of basal OCR level, 1.2 $\mu \mathrm{M}$ oligomycin (ATP synthase inhibitor), $4 \mu \mathrm{M}$ FCCP (proton gradient uncoupler), and $10 \mu \mathrm{M}$ antimycin $\mathrm{A}$ (mitochondria electron transport inhibitor) were sequentially added to assess their effects on OCR. Cell density and working concentrations for analysis was optimized according to the manufacturer's manual. OCR was automatically calculated, recorded, and plotted by Seahorse XF24 software version 1.8.

MMP measurement. Cells were seeded at densities of $5 \times 10^{4}$ per well in a 96-well black plate with a transparent bottom. Culture media was removed and wells were replenished with $100 \mu 1$ PBS containing $500 \mathrm{nM}$ of MitoTracker Red FM (Invitrogen), a fluorescent dye that stains mitochondria of live cells as a function of $\mathrm{m} \Delta \Psi$. Cells were incubated for $30 \mathrm{~min}$ at $37^{\circ} \mathrm{C}$ in $5 \% \mathrm{CO}_{2}$, and then washed with $100 \mu \mathrm{l}$ of warmed PBS per well. Fluorescence remaining in each well was measured on a microplate reader using $594 \mathrm{~nm}$ excitation and $642 \mathrm{~nm}$ emission wavelengths.

Intracellular ROS production. To quantify intracellular ROS, cells were seeded at densities of $5 \times 10^{4} /$ well in a 96 -well black plate. Culture media with treating agents was removed and wells were replenished with $100 \mu \mathrm{l}$ PBS containing $10 \mu \mathrm{M}$ 

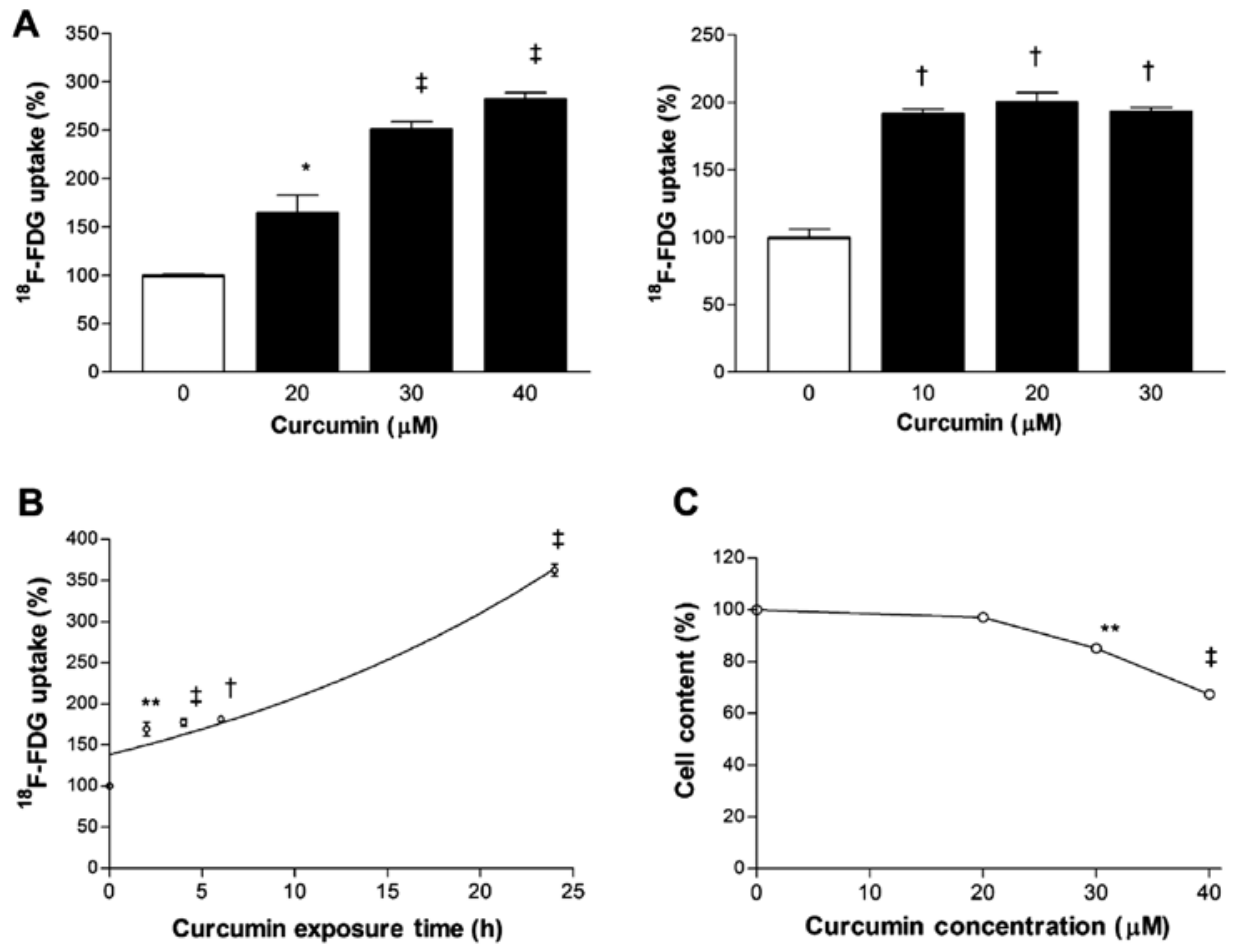

Figure 1. Curcumin augments cancer cell ${ }^{18} \mathrm{~F}$-FDG uptake. (A) Stimulation of ${ }^{18} \mathrm{~F}-\mathrm{FDG}$ uptake in MCF-7 breast cancer (left panel) and CT26 colon cancer cells (right panel) by 24 -h treatment with graded doses of curcumin. (B) Time course of ${ }^{18} \mathrm{~F}-\mathrm{FDG}$ uptake in MCF-7 cells stimulated by $30 \mu \mathrm{M}$ curcumin (C) Dose-dependent suppression of MCF-7 cell survival as assessed by SRB assays by 24 -h treatment with curcumin. All data are mean \pm SD $(n=3)$ expressed as \% controls. ${ }^{*} \mathrm{P}<0.05,{ }^{* *} \mathrm{P}<0.01,{ }^{\dagger} \mathrm{P}<0.0005,{ }^{\mathrm{P}} \mathrm{P}<0.0001$, compared to controls.

of CM- $\mathrm{H}_{2}$ DCFDA (Molecular Probes-Invitrogen), a cellpermeant fluorescent indicator for intracellular ROS. The probe is non-fluorescent until the acetate groups are removed by intracellular esterases and oxidation occurs within the cell, which can be detected by monitoring the increase in fluorescence. Cells were incubated with the probe for $30 \mathrm{~min}$ at $37^{\circ} \mathrm{C}$ in $5 \% \mathrm{CO}_{2}$, after which excessive dye was removed and cells were washed with $100 \mu \mathrm{l}$ of warmed PBS per well. Fluorescence remaining in each well was measured on a GloMax ${ }^{\circledR}$ microplate reader (Promega, Seoul, Korea) with $490 \mathrm{~nm}$ excitation and 510-570 nm emission wavelengths.

Western blot analysis. After cells were lysed with cold PRO-PREP lysis buffer (iNtRON Biotechnology, Seoul, Korea), $30 \mu \mathrm{g}$ of total cell lysate was separated by $10 \%$ SDS-PAGE and transferred to a polyvinylidene difluoride membrane. The membrane was blocked with 5\% non-fat milk in Tris-buffered saline with Tween-20 for $1 \mathrm{~h}$ at room temperature and incubated with primary antibodies $\left(1: 1,000 ; 4^{\circ} \mathrm{C}\right.$, overnight). The membrane was then incubated with HRP-linked anti-IgG (1:3,000; room temperature, $1 \mathrm{~h})$. Immunoreactive protein was detected by chemiluminescence, and band intensities were quantified on a GS-800 densitometer using Quantity One software (Bio-Rad Laboratories, Hercules, CA, USA).

Statistical analysis. All experiments were repeated two or three separate times, and the mean \pm SD of triplicate samples from a single representative experiment is presented unless otherwise specified. Student's t-tests were used to evaluate the statistical significance of measurements between groups, and P-values $<0.05$ were considered statistically significant.

\section{Results}

Curcumin dose-dependently stimulates cancer cell ${ }^{18} F-F D G$ uptake. Curcumin dose-dependently stimulated ${ }^{18} \mathrm{~F}-\mathrm{FDG}$ uptake of MCF-7 and CT26 cancer cells. Hence, ${ }^{18}$ F-FDG uptake corrected for cell content was substantially increased to $250 \pm 13$ and $193 \pm 5 \%$ of control levels by exposure to $30 \mu \mathrm{M}$ curcumin for MCF-7 cells and $10 \mu \mathrm{M}$ curcumin for CT26 cells for $24 \mathrm{~h}$ (Fig 1A). Time course experiments in MCF-7 cells disclosed that stimulation of ${ }^{18} \mathrm{~F}$-FDG uptake by $30 \mu \mathrm{M}$ curcumin began as soon as $2 \mathrm{~h}$, with further increases that reached $362.5 \pm 12.5 \%$ of control level by $24 \mathrm{~h}$ (Fig. 1B). This metabolic effect occurred at a curcumin dose that only mildly reduced surviving cell content $(85.1 \pm 2.7 \%$ of controls; Fig. 1C).

Curcumin stimulates glycolytic flux and suppresses mitochondrial respiration. In MCF-7 cells, treatment with $30 \mu \mathrm{M}$ curcumin for $24 \mathrm{~h}$ caused a significant elevation of lactate release to $144.6 \pm 3.9 \%$ of controls (Fig. 2A), consistent with a shift of metabolism toward glycolytic flux. To evaluate the contribution of the major mediators of cellular ${ }^{18} \mathrm{~F}-\mathrm{FDG}$ uptake, we measured hexokinase activity and membrane GLUT1 expression. As a result, $30 \mu \mathrm{M}$ curcumin for $24 \mathrm{~h}$ was found to significantly augment mitochondrial hexokinase activity to $146.0 \pm 34.5 \%$ of controls (Fig. 2B). In contrast, immunoblotting showed that curcumin had no significant influence on the level of membrane GLUT1 expression (data not shown).

We next evaluated whether the shift toward glycolytic flux by curcumin was associated with suppression of mitochondrial oxidative respiration. Extracellular flux analysis of MCF-7 cells demonstrated that treatment with $30 \mu \mathrm{M}$ curcumin for $6 \mathrm{~h}$ 
A

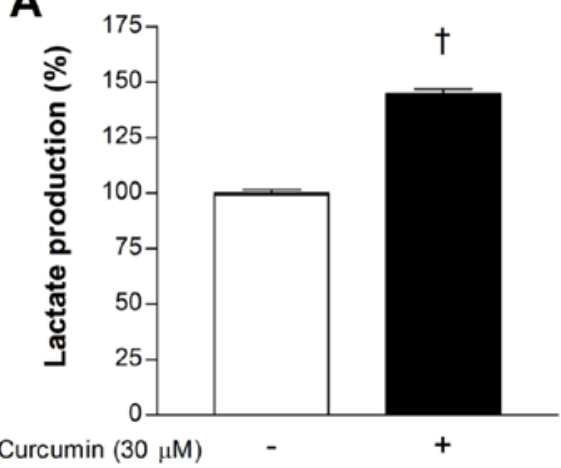

B

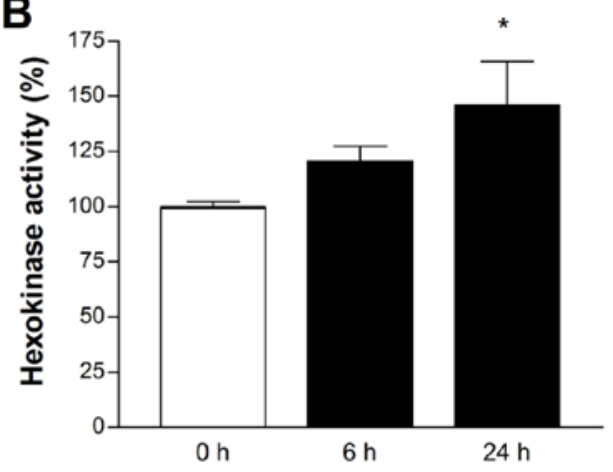

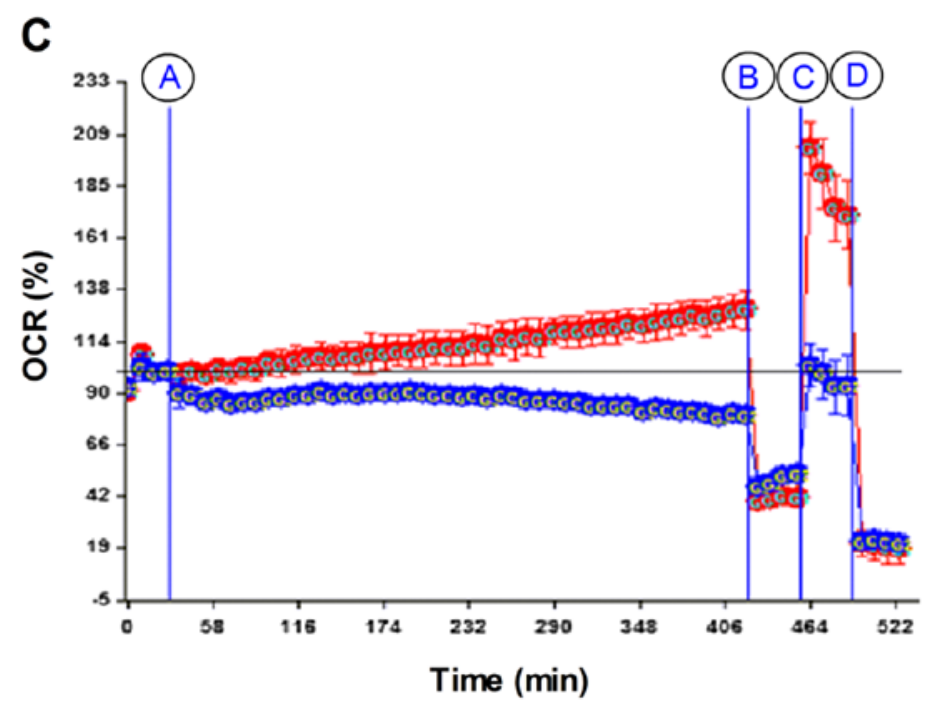

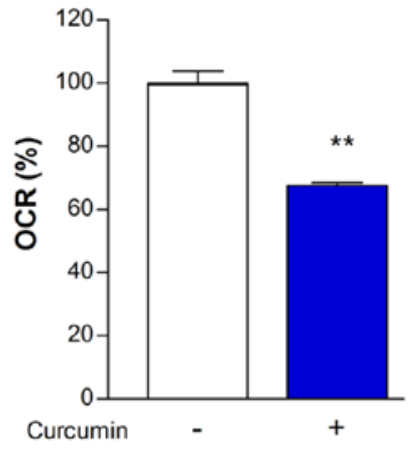

Figure 2. Curcumin shifts cancer cell metabolism toward glycolysis and suppresses mitochondrial oxidative respiration. (A and B) Stimulatory effects of $30 \mu \mathrm{M}$ curcumin on MCF-7 cell lactate production (A) and mitochondrial hexokinase activity (B). (C) OCR of MCF-7 cells at baseline and following treatment with vehicle (red) or $30 \mu \mathrm{M}$ curcumin (blue; point A). Points B, C and D indicate injection of oligomycin, FCCP and antimycin A, respectively (left panel). Mean OCR over $6 \mathrm{~h}$ following treatment with curcumin or vehicle is also shown (right panel). All bars are mean \pm SD ( $\mathrm{n}=3$ ) expressed as $\%$ controls. ${ }^{*} \mathrm{P}<0.05$, ${ }^{* *} \mathrm{P}<0.01,{ }^{\dagger} \mathrm{P}<0.0005$, compared to controls

caused a significant reduction of basal OCR to $67.5 \pm 1.4 \%$ of untreated controls (Fig. 2C). Inhibition of ATP synthase with oligomycin reduced OCR of control and curcumin-treated cells to similar levels, consistent with comparable amounts of proton leakage. Uncoupling of the proton gradient with FCCP caused a substantial augmentation of OCR for control cells but not for curcumin-treated cells. This indicates that maximal mitochondrial respiration is markedly suppressed by curcumin (Fig. 2C). Mean OCR over $6 \mathrm{~h}$ following treatment with curcumin was decreased to $67.6 \pm 0.4 \%$ of that following addition of vehicle (Fig. 2C).

Glycolysis inhibition with 2-DG potentiates the anticancer effect of curcumin. Given the shift toward glycolytic metabolism induced by curcumin, we next investigated whether additional blocking of glycolysis would potentiate its anticancer effect. 2-DG was first confirmed, as expected, to markedly diminished MCF-7 cell ${ }^{18} \mathrm{~F}-\mathrm{FDG}$ uptake. When used in combination, 2-DG also completely blocked the ability of curcumin to stimulate ${ }^{18} \mathrm{~F}$-FDG uptake. Hence, in the presence of $2-\mathrm{DG},{ }^{18} \mathrm{~F}-\mathrm{FDG}$ uptake was substantially reduced to $20.9 \pm 1.3 \%$ of untreated controls cells even when curcumin was added (Fig. 3A).
When antitumor effects were examined, curcumin and $2-\mathrm{DG}$ added separately caused only mild reductions of MCF-7 cell content to $87.1 \pm 2.9$ and $80.0 \pm 2.0 \%$ of controls, respectively. In comparison, co-treatment with curcumin and 2-DG reduced cell survival more efficiently to $67.1 \pm 1.9 \%$ of controls (Fig. 3B). We further evaluated how MAP kinase and mTOR signaling are affected by treatment. Immunoblotting revealed that curcumin, either alone or in combination with 2-DG, completely abrogated ERK activation (Fig. 3C). Activation of mTOR was not influenced by curcumin or 2-DG alone, but was significantly decreased to $52.5 \pm 11.1 \%$ of untreated control level when the two agents were combined (Fig. 3C).

Curcumin depolarizes cancer cell MMP and reduces intracellular ROS generation. To investigate how curcumin affects mitochondrial function, we measured MMP, which is critically involved in maintaining the mitochondrial respiratory chain and in avoidance of cell death. The results showed that exposure to curcumin for $24 \mathrm{~h}$ decreased MMP level of MCF-7 cells, in a manner inversely correlating to the applied dose. With a concentration of $30 \mu \mathrm{M}, \mathrm{MMP}$ dropped to $31.2 \pm 3.1 \%$ of control level (Fig. 4A). The reduction of MMP began as 

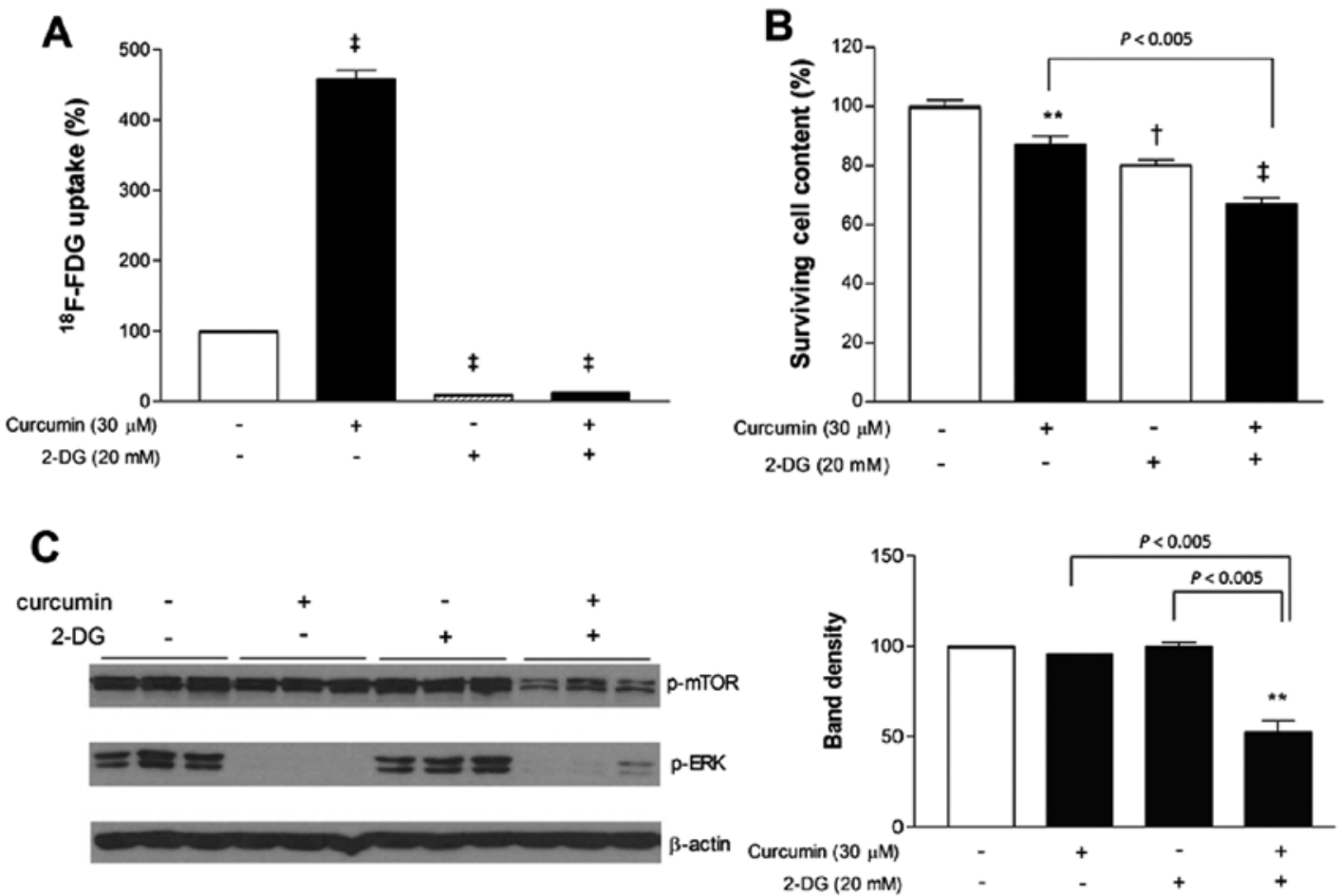

Figure 3. Effects of curcumin and 2-DG on cancer cell ${ }^{18} \mathrm{~F}$-FDG uptake and survival. (A and B) ${ }^{18} \mathrm{~F}-\mathrm{FDG}$ uptake (A) and surviving cell content (B) of MCF-7 cells treated for $24 \mathrm{~h}$ with vehicle (DMSO control) or $30 \mu \mathrm{M}$ curcumin, with or without glycolysis inhibition by $20 \mathrm{mM} 2$-DG. (C) Immunoblots of phosphorylated mTOR (p-mTOR) and phosphorylated ERK (p-ERK) in MCF-7 cells treated as above (left panel), and quantified relative p-mTOR band intensities (right panel). $\beta$-actin expression was used as loading control. Bars are mean $\pm \mathrm{SD}(\mathrm{n}=3)$ expressed as $\%$ of controls. ${ }^{* *} \mathrm{P}<0.01,{ }^{\dagger} \mathrm{P}<0.0005$, ${ }^{+} \mathrm{P}<0.0001$, compared to controls.
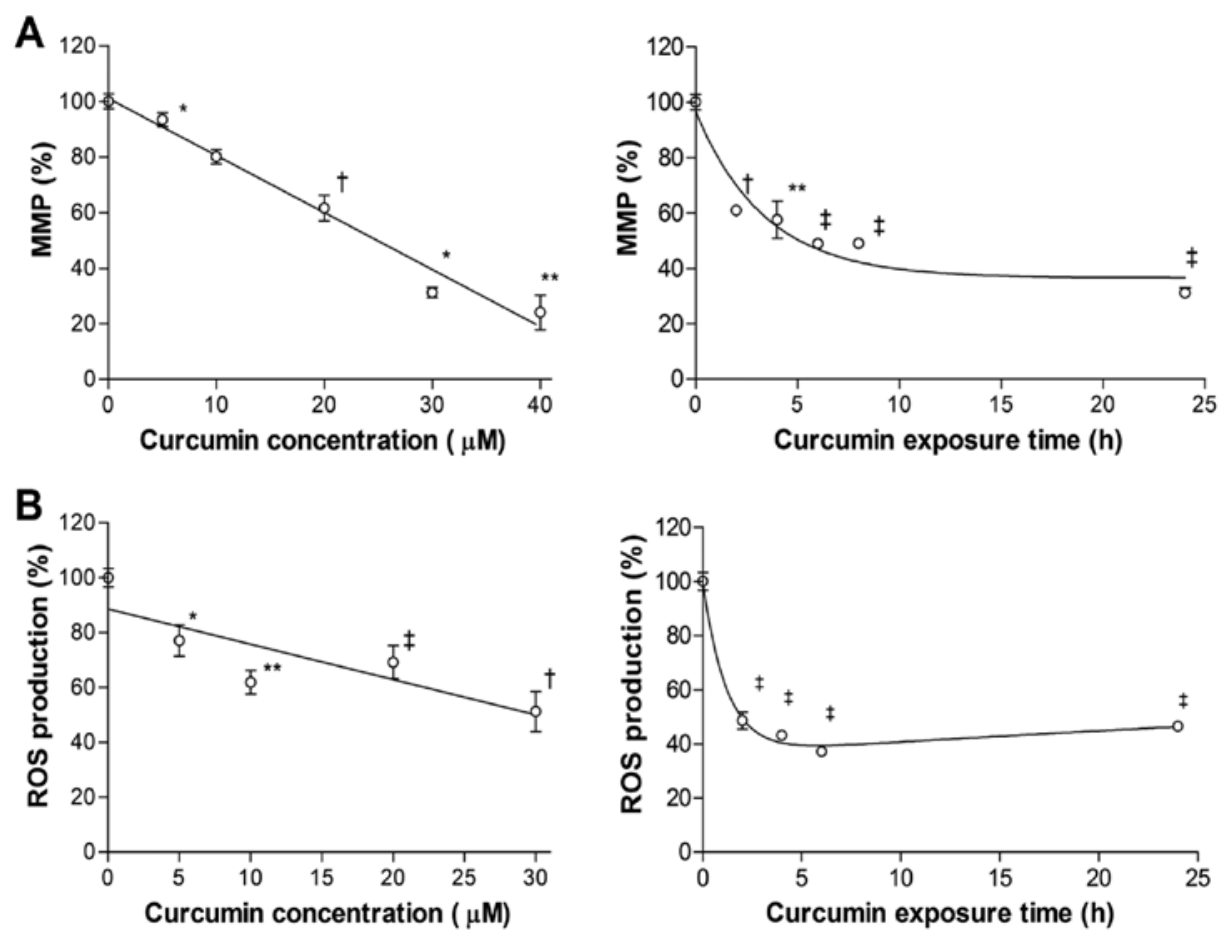

Figure 4. Curcumin suppresses MMP and ROS production. (A) Dose-dependent effects of treatment with curcumin on MCF-7 cell MMP (left panel), and time course of change in MMP following exposure to $30 \mu \mathrm{M}$ curcumin (right panel) $24 \mathrm{~h}$. (B) Dose-dependent effects of treatment with curcumin on MCF-7 cell ROS production (left panel), and time course of ROS production following exposure to $30 \mu \mathrm{M}$ curcumin (right panel) for $24 \mathrm{~h}$. All data are means $\pm \mathrm{SD}$ ( $\mathrm{n}=3$ ) expressed as \% controls. ${ }^{*} \mathrm{P}<0.05,{ }^{* *} \mathrm{P}<0.01,{ }^{\dagger} \mathrm{P}<0.0005,{ }^{\dagger} \mathrm{P}<0.0001$, compared to controls.

soon as $2 \mathrm{~h}$ of treatment and continued to decrease further over 24 h (Fig. 4A).
Intracellular ROS of MCF-7 cells were also dose-dependently reduced by curcumin, and the pattern was similar to the 
A

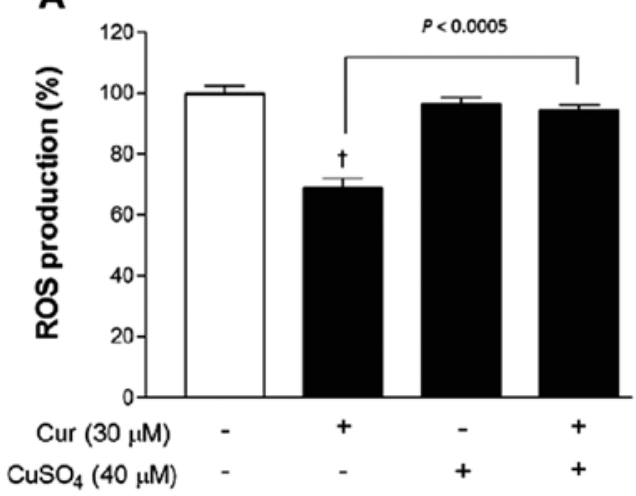

B

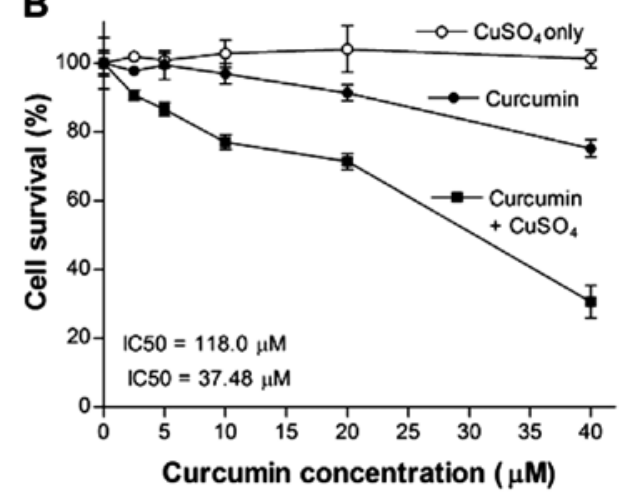

Figure $5 . \mathrm{CuSO}_{4}$ blocks the anti-oxidant effect and potentiates the anticancer effect of curcumin. (A) ROS production of MCF-7 cells treated for $4 \mathrm{~h}$ with $30 \mu \mathrm{M}$ curcumin in the presence or absence of $40 \mu \mathrm{M} \mathrm{CuSO}_{4}$. Bars are mean $\pm \mathrm{SD}(\mathrm{n}=3$ ) expressed as \% controls. (B) Surviving cell content following treatment with $\mathrm{CuSO}_{4}$, curcumin or curcumin plus $\mathrm{CuSO}_{4}$ for $24 \mathrm{~h}$. ${ }^{\dagger} \mathrm{P}<0.0005$, compared to controls.

decrease of MMP. Treatment with $30 \mu \mathrm{M}$ curcumin for $24 \mathrm{~h}$ reduced ROS level to $52.2 \pm 14.5 \%$ of controls (Fig. 4B). Time course experiments disclosed a rapid decline of ROS level to $48.6 \pm 6.2 \%$ of controls by $2 \mathrm{~h}$ (Fig. 4B). ROS decreased slightly further to $37.1 \pm 3.2 \%$ of controls by $6 \mathrm{~h}$, but slightly recovered thereafter to $46.5 \pm 3.4 \%$ of controls by $24 \mathrm{~h}$ (Fig. 4B).

Blocking the anti-oxidant property of curcumin markedly potentiates its anticancer effect. Finally, given the ROS-lowering property of curcumin in the literature as well as in our experimental data, we evaluated whether suppressing the anti-oxidant property of curcumin could enhance its anticancer effect. This was achieved by adding $40 \mu \mathrm{M} \mathrm{CuSO}_{4}$, which is reported to neutralize the anti-oxidant domains of curcumin. As a result, ROS level that was significantly reduced by $30 \mu \mathrm{M}$ curcumin alone, was recovered to control levels in the presence of copper (Fig. 5A).

SRB assays showed that $\mathrm{CuSO}_{4}$ alone caused no significant influence on MCF-7 cell survival in concentrations of up to $40 \mu \mathrm{M}$. However, when combined together, $40 \mu \mathrm{M} \mathrm{CuSO}_{4}$ substantially potentiated the dose-dependent reduction of cell survival induced by curcumin. Hence, cell content that was reduced to $75.2 \pm 2.6 \%$ of controls by $40 \mu \mathrm{M}$ curcumin alone was further reduced to $30.6 \pm 8.2 \%$ of controls in the presence of $40 \mu \mathrm{M} \mathrm{CuSO}_{4}$, pointing to a synergistic anticancer effect. Non-linear analysis of the dose-response curve showed $\mathrm{IC}_{50}$ values of 118.0 and $37.5 \mu \mathrm{M}$ for curcumin in the absence and presence of $\mathrm{CuSO}_{4}$, respectively (Fig. 5B).

\section{Discussion}

The present study study demonstrates that curcumin dosedependently stimulates ${ }^{18} \mathrm{~F}-\mathrm{FDG}$ uptake in MCF-7 breast cancer cells by shifting glucose metabolism toward glycolytic flux. This response is accompanied by a suppression of mitochondrial oxidative respiration and reduction of ROS production.

Curcumin is well-recognized for its anti-hyperglycemic effect through stimulation of glucose uptake in muscle tissue. However, little is known regarding its influence on glucose handling of malignant cells. Since curcumin is wellrecognized to exert anti-proliferative and apoptotic effects on breast cancer cells (17), we were particularly interested in how glucose metabolism and mitochondrial function of these cells are affected. Our results revealed that curcumin substantially enhances glucose uptake of both MCF-7 and CT26 cells, but MCF-7 cells showed a more robust dose-dependent response. As these two cell types are divergent in genetic makeup and biological property, it is difficult to point to a single reason explaining the different dose-responses of glucose uptake by curcumin. Nonetheless, this finding and our interest in breast cancer led us to select MCF-7 cells for further experiments. Although this study did not include normal cells, a previous study showed that curcumin and derivatives had minimal cytotoxic effects on normal mammary epithelial MCF-10A cells (18). In MCF-7 cells, the metabolic response began within a couple of hours of exposure, and was linked to stimulation of glycolytic flux, as evidenced by augmentations of lactate production and mitochondrial hexokinase activity. The significant reduction of OCR accompanying this response indicates a suppression of mitochondrial oxidative respiration. Curcumin further caused a marked blunting of the normal OCR response to FCCP, consistent with a severe reduction of maximal respiratory capacity. Tumor cells need to balance their metabolism in a manner that maintains a supply of energy production sufficient for continued growth and survival. Measurements of intracellular ATP would have strengthened our case but this was not performed in the present study. Taken together, our stimulation of glycolytic flux by curcumin appears to be a compensatory response of cancer cells attempting to maintain energy homeostasis in the face of mitochondrial dysfunction.

The dependence of malignant cells on a shift of energy metabolism to glycolytic flux to adapt to curcumin-induced suppression of mitochondrial respiration could provide a potential target for enhanced therapeutic efficacy. We therefore tested the effect of 2-DG, a glucose analogue that is taken up by transporters and competitively inhibits glycolysis at the phosphoglucoisomerase level (19). The results showed that stimulation of glycolysis by curcumin was completely blocked by 2 -DG. Importantly, this had a clear additive effect on reducing cell survival compared to that achieved by curcumin alone. This finding is reminiscent of a recent observation that mitochondria targeted cationic agents reduced cancer cell 
OCR and synergized with 2-DG to inhibit cancer cell proliferation and survival (20). Curcumin has the potential to directly or indirectly influence the biological activity of a number of signaling molecules, and its anti-proliferative effect on cancer cells may involve modulation of MAP kinase $(21,22)$ and mTOR pathways $(23,24)$. We therefore examined these pathways by western blotting, and found that curcumin near-completely suppressed ERK activation either alone or in combination with 2-DG. This finding is consistent with previous reports that link curcumin-mediated anti-proliferative actions to suppression of ERK activity. Guo et al (21) found in THP-1 acute monocytic leukemia cells that curcumin inhibited proliferation and induced apoptosis by blocking RAF/MEK/ERK and AKT/mTOR pathways. Furthermore, Xie et al (22) observed that curcumin inhibited nasopharyngeal carcinoma cell proliferation by reducing ERK1/2 expression. Interestingly, mTOR activation was unaffected by curcumin or 2-DG alone, but was significantly reduced by their combination. Curcumin has previously been shown to inhibit proliferation of colon cancer (23) and rhabdomyosarcoma cells (24) by mediating the inhibition of mTOR signaling. Furthermore, limiting energy availability of breast cancer cells by 2-DG has been shown to be accompanied by inhibition of mTOR activation (25). Taken together, our results suggest that blockade of mTOR signaling in cancer cells confronting mitochondrial dysfunction may contribute to the greater anti-proliferative effect observed by the combination of curcumin and 2-DG.

In addition to suppressing mitochondrial oxidative respiration, curcumin caused a dose-dependent decline of MMP that occurred within hours of treatment. A large amount of evidence indicates that mitochondrial are involved in the therapeutic properties of curcumin $(3,26)$. When mitochondria are damaged, there is an increase of proton leak that leads to diminished MMP, which in turn is an early event in the apoptotic process. Curcumin has previously been shown to exert depolarization and cause a collapse of MMP in hepatocellular cancer cells (27). Thus, the decline of MMP observed in our study supports a close link between the anticancer effect of curcumin and mitochondrial dysfunction.

The decrease of MMP by curcumin in our results was accompanied by dose- and time-dependent reductions of intracellular ROS. Mitochondria are a main source of cellular ROS, which is produced by electrons leaked from the mitochondrial respiratory chain during oxidative phosphorylation (8). It is generally accepted that ROS production has a positive relation with MMP level, and a decrease in MMP can reduce ROS production (28). Our observation of lowered ROS accompanying a decrease of MMP is therefore consistent with this notion. Nevertheless, the finding may seem puzzling given that elevated ROS plays a major role in the therapeutic effect of many anticancer agents (29). Furthermore, several lines of evidence indicate that it is the pro-oxidant, rather than their anti-oxidant, action of polyphenolic compounds that mediate their anticancer properties. It should be noted that curcumin is an excellent ROS scavenger that is bestowed with strong antioxidant activity under many circumstances. In fact, there are reports that cancer cells treated with curcumin display an antioxidant response that reduces ROS (11). Therefore, curcumin may have the capacity to act as either an anti-oxidant or a prooxidant in cancer cells, depending on the condition.
Curcumin can exhibit bifunctional anti-oxidant properties related to its ability to directly react with ROS and to indirectly induce expression of anti-oxidant proteins. Part of the curcumin molecule acts as chelator of positively charged metals, which play an important role in free radical trapping activity (2). A factor thought to play a major role in the exhibition of either anti-oxidant or pro-oxidant properties by curcumin is binding of metal ions. Based on our finding that ROS was lowered by curcumin, we examined whether addition of $\mathrm{Cu}^{2+}$ could enhance the pro-oxidant property, and thereby the anticancer effect, of the compound. The results revealed that when MCF-7 cells were simultaneously treated with $\mathrm{CuSO}_{4}$, cell content was more effectively decreased than by curcumin alone. This finding is consistent with a previous report of potentiated curcumin-induced cancer cell cytotoxicity by transient metals such as $\mathrm{Cu}^{2+}(30,31)$. Curcumin is composed of two hydrophobic phenyl domains connected by a $\beta$-diketone moiety, which acts as a binding site for transient metals (14). Copper, an essential trace metal with many physiological and pathological activities, is one of the most redox-active metal ions in living cells. Curcumin and $\mathrm{Cu}^{2+}$ have strong interactions, and their binding modifies the physico-chemical properties of the drug. In fact, mobilization of endogenous copper has been proposed to stimulate pro-oxidant action and contribute to the cytotoxic effects of several polyphenolic compounds against cancer (16).

In our results, intracellular ROS was no longer reduced by curcumin when $\mathrm{Cu}^{2+}$ was present. Similarly, a recent study showed that curcumin-induced cancer cell apoptosis was increased by $\mathrm{Cu}^{2+}$ through ROS generation, whereas it was blocked by anti-oxidants (31). These findings are consistent with the notion that, despite its anti-oxidant properties, curcumin can exhibit pro-oxidant effects in the presence of elevated $\mathrm{Cu}^{2+}$ (32). Indeed, increased ROS production was implicated in the DNA damage caused by DNA-associated copper and phenolic compounds (33). ROS-mediated DNA degradation was also observed in cancer cells treated with curcumin in the presence of $\mathrm{Cu}^{2+}(34,35)$. These findings have led to the exploration of curcumin or synthetic curcuminoids complexed with metal for enhanced antitumor activity (36). While metal-curcumin complexes may act either as antioxidants or pro-oxidants, curcumin complexed with $\mathrm{Cu}^{2+}$ has been shown to exert pro-oxidant effects by generating ROS at a high free copper level in a reducing environment (37). Taken together, our results suggest that $\mathrm{CuSO}_{4}$ blocks the direct ROS scavenging effect of curcumin.

In conclusion, curcumin treatment of MCF-7 cancer cells induces mitochondrial dysfunction that leads to a metabolic shift to glycolytic flux and reduced ROS generation. This leads to a significant increase in ${ }^{18} \mathrm{~F}-\mathrm{FDG}$ uptake, suggesting the possibility that it could serve as an imaging biomarker of tumor response to curcumin. Furthermore, glycolysis inhibition with 2-DG and suppression of the compound's antioxidant property with $\mathrm{Cu}^{2+}$ may enhance the anticancer action of curcumin.

\section{Acknowledgements}

The present study was supported by the Basic Science Research Program through the National Research Foundation 
of Korea (NRF) funded by the Ministry of Science, ICT \& Future Planning (grant no. 2014050612).

\section{References}

1. Anand P, Sundaram C, Jhurani S, Kunnumakkara AB and Aggarwal BB: Curcumin and cancer: An 'old-age' disease with an 'age-old' solution. Cancer Lett 267: 133-164, 2008.

2. Trujillo J, Granados-Castro LF, Zazueta C, Andérica-Romero AC, Chirino YI and Pedraza-Chaverrí J: Mitochondria as a target in the therapeutic properties of curcumin. Arch Pharm (Weinheim) 347: 873-884, 2014

3. Hanahan D and Weinberg RA: Hallmarks of cancer: The next generation. Cell 144: 646-674, 2011.

4. Zhao Y, Butler EB and Tan M: Targeting cellular metabolism to improve cancer therapeutics. Cell Death Dis 4: e532, 2013.

5. Larson SM and Schwartz LH: ${ }^{18}$ F-FDG PET as a candidate for 'qualified biomarker': Functional assessment of treatment response in oncology. J Nucl Med 47: 901-903, 2006.

6. Sullivan LB and Chandel NS: Mitochondrial reactive oxygen species and cancer. Cancer Metab 2: 17, 2014.

7. Cheng TC, Lin CS, Hsu CC, Chen LJ, Cheng KC and Cheng JT: Activation of muscarinic M-1 cholinoceptors by curcumin to increase glucose uptake into skeletal muscle isolated from Wistar rats. Neurosci Lett 465: 238-241, 2009.

8. Kim JH, Park JM, Kim EK, Lee JO, Lee SK, Jung JH, You GY, Park SH, Suh PG and Kim HS: Curcumin stimulates glucose uptake through AMPK-p38 MAPK pathways in L6 myotube cells. J Cell Physiol 223: 771-778, 2010.

9. Hail N Jr: Mitochondrial reactive oxygen species affect sensitivity to curcumin-induced apoptosis. Free Radic Biol Med 44 1382-1393, 2008.

10. Su CC, Lin JG, Li TM, Chung JG, Yang JS, Ip SW, Lin WC and Chen GW: Curcumin-induced apoptosis of human colon cancer colo 205 cells through the production of ROS, $\mathrm{Ca}^{2+}$ and the activation of caspase-3. Anticancer Res 26 (6B): 4379-4389, 2006.

11. Ak T and Gülçin I: Antioxidant and radical scavenging properties of curcumin. Chem Biol Interact 174: 27-37, 2008.

12. Kunwar A, Jayakumar S, Srivastava AK and Priyadarsini KI: Dimethoxycurcumin-induced cell death in human breast carcinoma MCF7 cells: Evidence for pro-oxidant activity, mitochondrial dysfunction, and apoptosis. Arch Toxicol 86: 603-614, 2012.

13. Li YB, Gao JL, Zhong ZF, Hoi PM, Lee SM and Wang YT: Bisdemethoxycurcumin suppresses MCF-7 cells proliferation by inducing ROS accumulation and modulating senescence-related pathways. Pharmacol Rep 65: 700-709, 2013.

14. Priyadarsini KI: The chemistry of curcumin: From extraction to therapeutic agent. Molecules 19: 20091-20112, 2014.

15. Verma SP and Goldin BR: Copper modulates activities of genistein, nitric oxide, and curcumin in breast tumor cells. Biochem Biophys Res Commun 310: 104-108, 2003.

16. Hadi SM, Asad SF, Singh S and Ahmad A: Putative mechanism for anticancer and apoptosis-inducing properties of plant-derived polyphenolic compounds. IUBMB Life 50: 167-171, 2000.

17. Liu D and Chen Z: The effect of curcumin on breast cancer cells. J Breast Cancer 16: 133-137, 2013.

18. Reddy CA, Somepalli V, Golakoti T, Kanugula AK, Karnewar S, Rajendiran K, Vasagiri N, Prabhakar S, Kuppusamy P, Kotamraju S, et al: Mitochondrial-targeted curcuminoids: A strategy to enhance bioavailability and anticancer efficacy of curcumin. PLoS One 9: e89351, 2014.

19. Wick AN, Drury DR, Nakada HI and Wolfe JB: Localization of the primary metabolic block produced by 2-deoxyglucose. J Biol Chem 224: 963-969, 1957.

20. Cheng G, Zielonka J, McAllister D, Hardy M, Ouari O, Joseph J, Dwinell MB and Kalyanaraman B: Antiproliferative effects of mitochondria-targeted cationic antioxidants and analogs: Role of mitochondrial bioenergetics and energy-sensing mechanism. Cancer Lett 365: 96-106, 2015.
21. Guo Y, Shan Q, Gong Y, Lin J, Shi F, Shi R and Yang X Curcumin induces apoptosis via simultaneously targeting AKT/ mTOR and RAF/MEK/ERK survival signaling pathways in human leukemia THP-1 cells. Pharmazie 69: 229-233, 2014.

22. Xie YQ, Wu XB and Tang SQ: Curcumin treatment alters ERK-1/2 signaling in vitro and inhibits nasopharyngeal carcinoma proliferation in mouse xenografts. Int J Clin Exp Med 7: 108-114, 2014.

23. Johnson SM, Gulhati P, Arrieta I, Wang X, Uchida T, Gao T and Evers BM: Curcumin inhibits proliferation of colorectal carcinoma by modulating Akt/mTOR signaling. Anticancer Res 29: 3185-3190, 2009.

24. Beevers CS, Li F, Liu L and Huang S: Curcumin inhibits the mammalian target of rapamycin-mediated signaling pathways in cancer cells. Int J Cancer 119: 757-764, 2006.

25. Jiang W, Zhu Z and Thompson HJ: Modulation of the activities of AMP-activated protein kinase, protein kinase B, and mammalian target of rapamycin by limiting energy availability with 2-deoxyglucose. Mol Carcinog 47: 616-628, 2008.

26. Wu SH, Hang LW, Yang JS, Chen HY, Lin HY, Chiang JH, Lu CC Yang JL, Lai TY, Ko YC, et al: Curcumin induces apoptosis in human non-small cell lung cancer NCI-H460 cells through ER stress and caspase cascade- and mitochondria-dependent pathways. Anticancer Res 30: 2125-2133, 2010.

27. Cao J, Liu Y, Jia L, Zhou HM, Kong Y, Yang G, Jiang LP Li QJ and Zhong LF: Curcumin induces apoptosis through mitochondrial hyperpolarization and mtDNA damage in human hepatoma G2 cells. Free Radic Biol Med 43: 968-975, 2007.

28. Suski JM, Lebiedzinska M, Bonora M, Pinton P, Duszynski J and Wieckowski MR: Relation between mitochondrial membrane potential and ROS formation. Methods Mol Biol 810: 183-205, 2012.

29. Gorrini C, Harris IS and Mak TW: Modulation of oxidative stress as an anticancer strategy. Nat Rev Drug Discov 12: 931-947, 2013.

30. Lou JR, Zhang XX, Zheng J and Ding WQ: Transient metals enhance cytotoxicity of curcumin: Potential involvement of the NF-kappaB and mTOR signaling pathways. Anticancer Res 30: 3249-3255, 2010

31. Lu JJ, Cai YJ and Ding J: The short-time treatment with curcumin sufficiently decreases cell viability, induces apoptosis and copper enhances these effects in multidrug-resistant K562/A02 cells. Mol Cell Biochem 360: 253-260, 2012.

32. Urbina-Cano P, Bobadilla-Morales L, Ramírez-Herrera MA, Corona-Rivera JR, Mendoza-Magaña ML, Troyo-Sanromán R and Corona-Rivera A: DNA damage in mouse lymphocytes exposed to curcumin and copper. J Appl Genet 47: 377-382, 2006.

33. Li Y and Trush MA: Reactive oxygen-dependent DNA damage resulting from the oxidation of phenolic compounds by a copperredox cycle mechanism. Cancer Res 54 (Suppl): 1895s-1898s, 1994.

34. Ahsan H and Hadi SM: Strand scission in DNA induced by curcumin in the presence of $\mathrm{Cu}(\mathrm{II})$. Cancer Lett 124: 23-30, 1998.

35. Ahsan H, Parveen N, Khan NU and Hadi SM: Pro-oxidant, anti-oxidant and cleavage activities on DNA of curcumin and its derivatives demethoxycurcumin and bisdemethoxycurcumin. Chem Biol Interact 121: 161-175, 1999.

36. John VD, Kuttan G and Krishnankutty K: Anti-tumour studies of metal chelates of synthetic curcuminoids. J Exp Clin Cancer Res 21: 219-224, 2002 .

37. Leung MH, Harada T and Kee TW: Delivery of curcumin and medicinal effects of the copper(II)-curcumin complexes. Curr Pharm Des 19: 2070-2083, 2013. 